$\begin{array}{ll}\text { Research Square } & \begin{array}{l}\text { Preprints are preliminary reports that have not undergone peer review. } \\ \text { They should not be considered conclusive, used to inform clinical practice, } \\ \text { or referenced by the media as validated information. }\end{array}\end{array}$

\title{
Acupuncture For Major Depressive Disorder: A Systematic Review and Meta-Analysis of High-Quality Randomized Control Trials
}

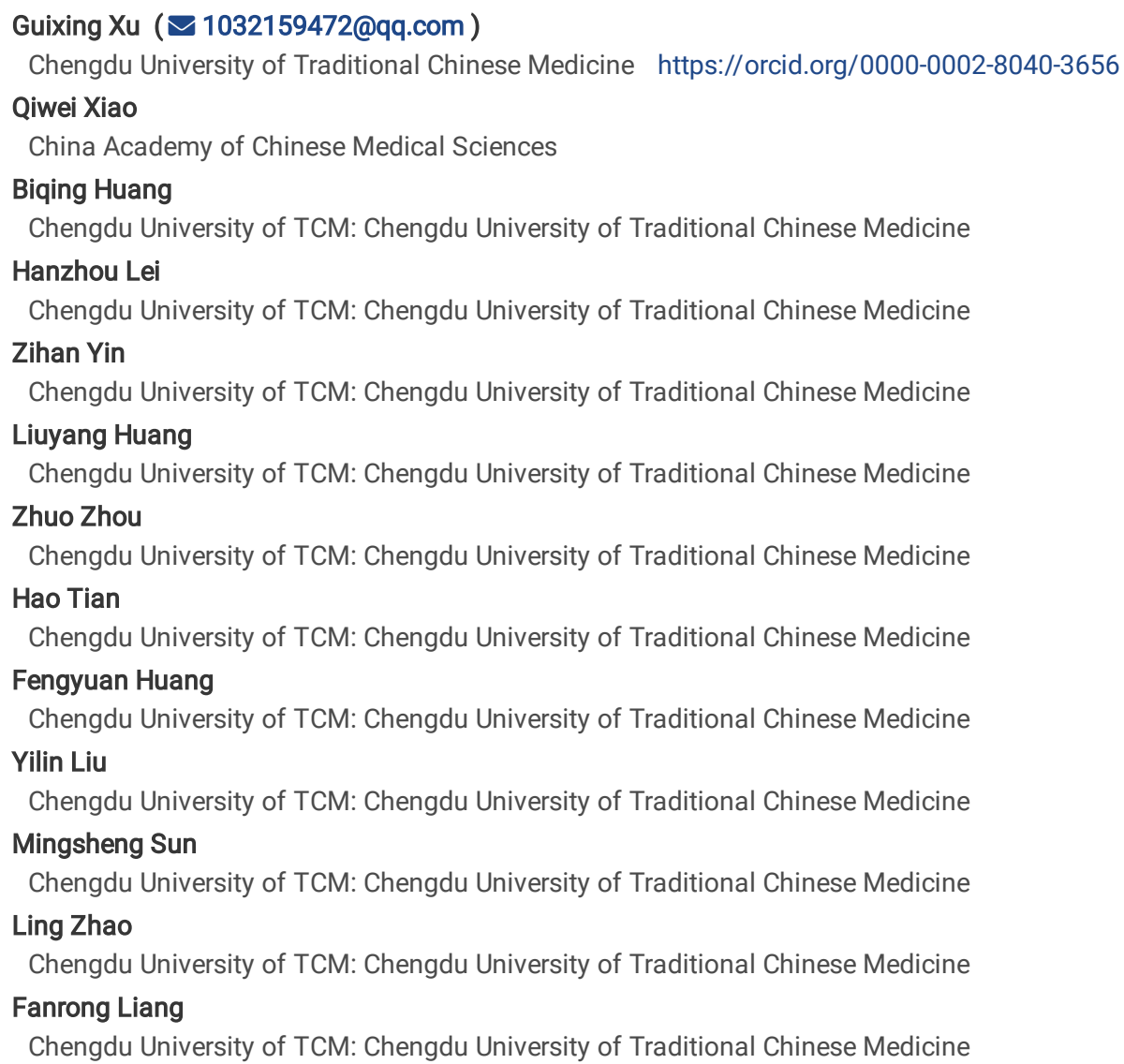

\section{Research}

Keywords: Acupuncture, depression, Systematic review, Meta-analysis, Effectiveness, Safety

Posted Date: November 16th, 2021

DOl: https://doi.org/10.21203/rs.3.rs-1064326/v1

License: (a) (1) This work is licensed under a Creative Commons Attribution 4.0 International License. Read Full License 


\section{Abstract}

Background: The effects of acupuncture for major depressive disorder (MDD) uncertain. This review aims to determine the effects of Acupuncture for MDD .

Methods: Eight Database were searched to identify randomized control trials (RCTs) on Acupuncture for MDD. All RCTs with adult participants undergoing acupuncture treatment for MDD were included. The primary outcome measure was the 24-item Hamilton rating scale for depression (HAMD-24). We used random-effects meta-analysis to synthesis the results with mean difference or odds ratio. Furthermore, the potential heterogeneity was tested through meta-regression/subgroup analyses/sensitive analysis. The quality of evidence for each outcome was assessed by the Grading of Recommendations Assessment, Development and Evaluation approach.

Results: Forty-three studies were included: 9 acupuncture versus sham-acupuncture ( $n=920), 26$ acupuncture versus antidepressants ( $n=2169), 9$ acupuncture plus antidepressants versus antidepressants ( $n=667)$. Of the 43 high-quality articles, 24 and 8 were determined to have a low and moderate risk of bias, respectively. The pooled results for HAMD-24 and SDS revealed the clinical benefits of Acupuncture or Acupuncture plus antidepressants compared to sham-acupuncture or antidepressants, with high quality evidence. Furthermore, high quality of evidence showed that acupuncture led to fewer adverse effects compared to antidepressants.

Conclusions: Acupuncture or acupuncture plus antidepressants were significantly associated with reduced HAMD-24 scores, with high-quality evidence. More rigorous trials are needed to identify the optimal frequency of Acupuncture for MDD and integrate such evidence into clinical care to reduce antidepressant use.

\section{Strengths And Limitations Of This Study}

1. The Jadad scores of the original studies included in this study were all greater than or equal to 4 , ensuring the credibility and high quality of the study results.

2. This study used the GRADE Guidelines to assess the quality of evidence of the meta-analysis with high quality.

3. The results of this study will be useful to physicians in clinical decision-making and will clarify the effectiveness and safety of acupuncture in treating MDD.

\section{Background}

Major depressive disorder (MDD) is a common psychological condition with an estimated lifetime prevalence of $16 \%$, affecting more than 320 million people across the globe ${ }^{1}$. As of April 2017, depression has been recognized by the World Health Organization (WHO) as the leading cause of health-related disability, accounting for approximately $4.4 \%$ of all disabilities and premature deaths worldwide 2 .

Antidepressants such as selective serotonin reuptake inhibitors or selective serotonin-norepinephrine reuptake inhibitors are commonly used to treat MDD. Most evidence-based guidelines recommend antidepressants to be the first-line therapy because of their favorable outcomes and superior characteristics, including broad-spectrum effectiveness, safety, tolerability, simplicity of use, and low cost $^{3}$. Nevertheless, pharmacological interventions also have undesirable side effects, including central nervous system and gastrointestinal disorders, weight gain, sexual dysfunction, and adverse emotional effects ${ }^{4}$. In addition, long-term use may also cause drug tolerance, withdrawal symptoms when discontinued and increased suicidal ideation in certain patient populations ${ }^{5}$.

As an essential component of traditional Chinese medicine, acupuncture therapy has been practiced in China for thousands of years in disease prevention and treatment, functional improvement, longevity enhancement, and regulating emotions. As early as 1979, the WHO held a symposium on Acupuncture and created a list of 43 diseases suitable for Acupuncture ${ }^{6}$. In 2002, the WHO recommended Acupuncture as a treatment for depression (including depressive neurosis and depression following stroke) ${ }^{7}$. However, significant heterogeneity has been reported in findings of several randomized control trials (RCTs) ${ }^{8-14}$.

Clinical trials have demonstrated that the effect of acupuncture therapy was partly induced via the autonomic nervous system ${ }^{15-17}$. In this regard, manual acupuncture leads to a wide range of central nervous system responses involving the amygdala, hippocampus, hypothalamus, cerebellum and other limbic structures, documented by functional magnetic resonance imaging and EEG ${ }^{17}$. Moreover, animal studies have shown that acupuncture therapy exerts its effects via multi-receptor and multi-pathway regulation related to amino acid metabolism and in $\square$ ammatory pathways, especially the Toll-like receptor signaling pathway, tumor necrosis factor signaling pathway and the nuclear factor kappa-light-chain enhancer of activated B cells (NF-kB) signaling pathway ${ }^{18}$. Besides, acupuncture therapy has been reported to influence the neurotransmitter levels of serotonin and noradrenaline and the adenylate cyclase cyclic adenosine monophosphate-protein kinase A cascade within the central nervous system via mechanisms similar to antidepressant medication ${ }^{19}$.

Although several systematic reviews and meta-analyses suggested that acupuncture therapy effectively treated MDD ${ }^{20-26}$, some review of these studies found some quality issues, and the original studies did not yield consistent results ${ }^{20-22}$. Therefore, we conducted this meta-analysis, including only high- 


\section{Methods}

\subsection{Study registering and reporting}

This protocol was registered on INPLASY International Prospective Register of Systematic Reviews (INPLASY2021100073). It was conducted in accordance with the Measure Tool to Assess Systematic Reviews (AMSTAR 2) ${ }^{23}$, and the final report was presented following the Preferred Reporting Items for Systematic Reviews and Meta-Analyses (PRISMA) statements ${ }^{2425}$.

\subsection{Selection criteria}

\section{Study design}

We included all RCTs with eligible intervention(s) and outcome(s) for MDD published in Chinese and English. For the crossover trials with the randomized control design, the first stage consisted of data collection for this meta-analysis. Furthermore, we only included RCTs with a Jadad score $\geq 4$ since the previously published systematic review could not obtain accurate conclusions due to the low quality of RCTs included.

\section{Population}

Patients (aged $\geq 18$ years) were diagnosed with MDD according to the third edition of Chinese Classification of Mental Disorders (CCMD-2/3) ${ }^{26}$, the International Statistical Classification of Diseases and Related Health Problems (ICD-10/11) 27 , or the Diagnostic and Statistical Manual of Mental Disorders (DSM-II/III/III-R/IV/IV-TR/V) ${ }^{28} 29$.

\section{Interventions/Comparators}

The experimental group consisted of patients that received Acupuncture or Acupuncture plus antidepressants. The acupoint numbers, retaining time and frequency, treatment sessions were not limited.

The control group included non-acupuncture techniques, such as placebo control or other active therapies.

\section{Outcome measures}

The primary outcome was the Hamilton Depression Rating Scale for Depression (HAMD17/24). The secondary outcomes included changes in the Zung Self-rating Depression Scale (SDS) and the incidence of adverse effects.

The data at each time point was extracted from the original trials to be analyzed in this study.

\subsection{Literature search}

The following online databases were searched: EMBASE, The Cochrane Library, PubMed, The Cochrane Central Register of Controlled Trials (CENTRAL), Web of Science, The China National Knowledge Infrastructure (CNKI), The Wanfang database, The VIP Database for Chinese Technical Periodicals, The China Doctoral Dissertations Full-text Database (CDFD). All RCTs were published from inception to 10th September 2021. The Cochrane library database search strategy is shown in Appendix 1

\subsection{Data collection and analysis}

\subsubsection{Selection of studies}

The process of data screening and study selection is displayed in Figure 1. First, studies were imported into Endnote X9 to remove duplicates, including duplications from different publications and multilingual publications and reports on different aspects of the same research. Two investigators (XGX and HBQ) independently assessed the titles and abstracts of the articles to select eligible studies based on the inclusion criteria. Subsequently, two other inves tigators (HLY and XQW) performed full-text assessments to exclude articles according to the exclusion criteria. Points of disagreement were resolved through consensus or by consulting a fifth investigator (LFR).

\subsubsection{Risk of bias and quality assessment}

The risk of bias of eligible trials was measured by the Risk of Bias (ROB) Tool in Cochrane Handbook (5.1.0) ${ }^{30}$ by two independent researchers (HFY and LYL). Each criterion was graded as "low" risk of bias, "high" risk of bias and "unclear" risk of bias. The methodology quality was assessed by two independent investigators (XQW and HBQ) with the Jadad scale. Any dissent occured in the assessment procedures was judged by a third investigator (SMS).

\subsubsection{Data extraction}


First, a standard table for electronic data extraction was established during a general meeting. After crosschecking for duplicates, the data were extracted by two authors (ZZ and TH) independently. The collected data included: basic information of studies (Study ID, the published date, author information, title, publication); characteristic of trials (study design, sample size, grouping method, blinding, assessment of outcomes, objectives of the study, etc.); the participants (age, gender, ethnicity, country, diagnosis, duration); method of intervention/control (number of treatment, frequency, duration of a session, additional treatment, etc.); outcome measurements (primary outcome, secondary outcome, assessment timeline, length of follow-up, etc.); results (mean, $\mathrm{SD}$, adverse event, etc.). In addition, the extracted data was saved in an Excel format, and a third researcher (XQW) crosschecked the data input to ensure consistency and validity.

\subsubsection{Unification of data and dealing with missing data}

Before statistical analysis, the unit and time of extracted data were unified to ensure the accuracy of the data analysis. For studies with incomplete data, our researchers invested time and effort to contact the first author or corresponding author to collect the incomplete data as far as possible. If the data was not obtained, a systematic review will be conducted for these data instead of statistical synthesis.

\subsubsection{Data synthesis}

Meta-analysis of RCTs with available data was performed by calculating the effect size and $95 \% \mathrm{Cl}$ using the random-effects model. Heterogeneity among trials was identified by the $\chi^{2}$ test and reported as $\mathrm{I}^{2}$. Statistical analyses were performed with RevMan 5.2 and Stata 15.0. Two-sided P-value $<0.05$ was considered statistically significant.

Studies were grouped according to the type of intervention (acupuncture, Acupuncture plus antidepressants) and the controls (sham-acupuncture, antidepressants, and sham-acupuncture plus antidepressants). For studies with more than 1 control group, such as Acupuncture versus sham acupuncture versus antidepressants, the results were split into pairwise comparisons by the different comparators.

Given the reported strong correlation between HAMD-17 and HAMD-24, the HAMD-17 scores were converted to the corresponding HAMD-24 scores.

Meta-regression and sensitivity analyses were conducted to explore potential sources of heterogeneity. Meta-regression was used to explore whether the age of patients, baseline HAMD-24, course of MDD, and acupuncture session affect the effectiveness of Acupuncture. Sensitivity analysis was used to identify studies that significantly affected the overall effect.

Publication bias was assessed by funnel plots for asymmetry when at least ten trials were included.

Evidence quality evaluation: The quality of evidence for the outcomes was assessed with the Grading of Recommendations Assessment, Development, and Evaluations (GRADE) system ${ }^{31}$ and rated as high, moderate, low, and very low. A summary of findings is presented in Table 1.

Table 1

GRADE quality assessment of evidence

\begin{tabular}{|c|c|c|c|c|c|c|c|c|c|c|}
\hline Intervention & $\begin{array}{l}\text { Outcome } \\
\text { indicators }\end{array}$ & $\begin{array}{l}\text { Risk } \\
\text { of } \\
\text { bias }\end{array}$ & Inconsistency & Indirectness & Imprecision & $\begin{array}{l}\text { Publication } \\
\text { bias }\end{array}$ & $\begin{array}{l}\text { Large } \\
\text { effect }\end{array}$ & $\begin{array}{l}\text { Dose- } \\
\text { response }\end{array}$ & $\begin{array}{l}\text { All plausible } \\
\text { confounding }\end{array}$ & $\begin{array}{l}\text { Quality } \\
\text { of } \\
\text { evidence }\end{array}$ \\
\hline \multirow{3}{*}{$\begin{array}{l}\text { acupuncture } \\
\text { versus sham- } \\
\text { acupuncture }\end{array}$} & HAMD-24 & 0 & $-7^{b}$ & 0 & 0 & 0 & 0 & 0 & 0 & High \\
\hline & SDS & 0 & $-1^{\mathrm{b}}$ & 0 & $-1^{c}$ & 0 & 0 & 0 & 0 & Moderate \\
\hline & $\begin{array}{l}\text { Side } \\
\text { effect }\end{array}$ & 0 & $-1^{b}$ & 0 & $-1^{c}$ & $-1^{d}$ & 0 & 0 & 0 & Low \\
\hline \multirow{3}{*}{$\begin{array}{l}\text { acupuncture } \\
\text { versus } \\
\text { antidepressants }\end{array}$} & HAMD-24 & 0 & $-1^{b}$ & 0 & 0 & 0 & 0 & 0 & 0 & High \\
\hline & SDS & 0 & $-1^{b}$ & 0 & 0 & 0 & 0 & 0 & 0 & High \\
\hline & $\begin{array}{l}\text { Side } \\
\text { effect }\end{array}$ & 0 & 0 & 0 & 0 & $-1^{d}$ & 0 & 0 & 0 & High \\
\hline \multirow{4}{*}{$\begin{array}{l}\text { acupuncture } \\
\text { plus } \\
\text { antidepressants } \\
\text { versus } \\
\text { antidepressants }\end{array}$} & HAMD-24 & $-1^{a}$ & 0 & 0 & 0 & 0 & 0 & 0 & 0 & High \\
\hline & SDS & 0 & $-1^{\mathrm{b}}$ & 0 & $-1^{c}$ & $-1^{d}$ & 0 & 0 & 0 & Low \\
\hline & $\begin{array}{l}\text { HAMD-24 } \\
\text { in follow- } \\
\text { up }\end{array}$ & $-1^{a}$ & 0 & 0 & 0 & $-1^{d}$ & 0 & 0 & 0 & Moderate \\
\hline & $\begin{array}{l}\text { Side } \\
\text { effect }\end{array}$ & 0 & $-1^{b}$ & 0 & $-1^{c}$ & $-1^{d}$ & 0 & 0 & 0 & Low \\
\hline
\end{tabular}

\section{Results}




\subsection{Description of included studies}

The initial electronic search yielded 9943 unique records. 296 RCTs on Acupuncture for MDD were selected. Screening and full-text article analysis identified 43 high-quality RCTs with Jadad score $\geq 4$, including 4037 patients (Figure 1, appendix 2) comparing acupuncture/acupuncture plus antidepressants versus sham-acupuncture/antidepressants. Appendix 3 shows the Jadad score in included trials (Jadad $\geq 4$ ), while Appendix 4 presents the Jadad score in excluded trials (Jadad<4). The sample size in the included trials ranged from 20 to 176, with a mean age of 41.46 (range 30-52.33) and included 1466 men. At the baseline, the mean HAMD-24 score was 32.27 (range 18.2-58.14). Eligible RCTs included one study from India ${ }^{8}$ and forty-two from China ${ }^{9-13} 32-68$. Qualitative synthesis was conducted in two studies ${ }^{10} 43$ due to lack of raw data, and quantitative synthesis in forty-one studies ${ }^{8} 11$ 32-42 44-57

\subsection{Risk of bias assessment}

Appendix 5 presents the risk of bias in each trial. One major limitations of this study was low levels of blinding reported for participants, investigators, and outcome assessors $^{3233363841-4547-50525861626466-68}$. Forty-three (100\%) trials ${ }^{8-1332-68}$ had low risk of bias in random sequence generation while 22 $(51.2 \%)^{9} 11-1332-35414246475154-56586062636568$ had low risk of bias in allocation concealment. Ten RCTs out of $43(23.3 \%)^{891112405155-5765}$ reported blinding for participants and investigators, and 9 RCTs (20.9\%) ${ }^{91112405155-5765}$ reported blinding for outcome assessment. Thirty-eight trials (88.4\%) ${ }^{8-11}$ 13323335-4244-5052-5456-68 were adjudicated as being at low risk of attrition bias, 15 trials ${ }^{113345464856575961-6668}$ (34.9\%) were at low risk of bias from selective outcome reporting, and 4 trials ${ }^{11596365}(9.3 \%)$ were at low risk of bias from other bias.

\subsection{Outcomes}

\subsubsection{HAMD-24}

Meta-analysis of 6 trials yielded a significant difference in favor of acupuncture (MD: -4.49 [95\% Cl: -6.41 to -2.15$]$; $\left.\mathrm{I}^{2}=76 \%\right)$ compared with sham acupuncture, with high-quality evidence (Figure 2 and Table 1). Pooled results from 24 RCTs showed that acupuncture (MD: -2.17 [95\% Cl: -3.31 to -1.03]; $\mathrm{I}^{2}=85 \%$ ) was more effective than antidepressants, while the funnel plot showed no publication bias, with high-quality evidence (Figure3, Figure4, and Table 1). High-quality evidence from 9 studies showed that acupuncture plus antidepressants (MD: -5.34 [95\% $\mathrm{Cl}:-6.68$ to -4.00$] ; \mathrm{I}^{2}=38 \%$ ) had more benefits in improving MDD (Figure5 and Table 1). Analysis of follow-up outcomes showed that treatment with acupuncture plus antidepressants was more effective than with antidepressants alone, with moderate-quality evidence (Table 1 and Appendix 6).

During the meta-regression analysis of acupuncture versus sham-acupuncture, we found that the disease course of MDD could significantly reduce the heterogeneity (adjusted R2 = 51.87\%, I2resid = 60.27\%, P=0.039, Figure 6). Meta-regression for age (adjusted $\mathrm{R}^{2}:-15.65 \%, \mathrm{I}^{2}$ resid: $79.01 \%, \mathrm{P}: 0.906$ ), baseline HAMD-24 (adjusted $R^{2}: 17.05 \%, I^{2}$ resid: $72.66 \%, P: 0.181$ ), and acupuncture session (adjusted $R^{2}: 34.67 \%, I^{2}$ resid: 66.62\%, P: 0.095) were not statistically significant (Appendix 7). During the comparison between acupuncture versus antidepressants, the baseline HAMD-24 could reduce the heterogeneity (adjusted $\mathrm{R}^{2}=41.96 \%, \mathrm{I}_{\text {resid }}^{2}=75.13 \%, \mathrm{P}=0.001$ ) (Figure 7). Furthermore, meta-regression for age (adjusted $\mathrm{R}^{2}: 4.56 \%, \mathrm{I}^{2}$ resid: $82.02 \%, \mathrm{P}: 0.157$ ), disease course (adjusted $\mathrm{R}^{2}:-3.28 \%, \mathrm{I}^{2}$ resid: $82.64 \%, \mathrm{P}: 0.531$ ), and acupuncture session (adjusted $\mathrm{R}^{2}:-1.73 \%, \mathrm{I}^{2}$ resid: $83.52 \%, \mathrm{P}: 0.386$ ) were not statistically significant (Appendix 8).

During the sensitivity analyses of Acupuncture versus sham acupuncture and Acupuncture versus antidepressants, no studies with significant differences were found (Appendix 9 and Appendix 10). However, for acupuncture plus antidepressants versus antidepressants, the heterogeneity significantly decreased (MD: -5.34 [95\% Cl: -6.68 to -4.00 ]; $\mathrm{I}^{2}=38 \%$ ) to (MD: $-4.83\left[95 \% \mathrm{Cl}:-5.89\right.$ to -3.77 ]; $\mathrm{I}^{2}=0 \%$ ) (Figure 8).

\subsubsection{SDS}

Pooled analysis of four studies showed that Acupuncture (MD: -8.54 [95\% Cl: -13.01 to -4.06$]$; $I^{2}=81 \%$ ) was more effective than sham acupuncture, with moderate quality of evidence (Table 1 and Appendix 11). Furthermore, high-quality evidence from 10 RCTs showed that Acupuncture and antidepressants were of equal effectiveness for MDD but was potentially influenced by publication bias (Table 1, Appendix 12 and Appendix 13). Moreover, pooled results from 4 RCTs provided low-quality evidence that Acupuncture plus antidepressants were not as beneficial as antidepressants for MDD treatment (Table 1 and Appendix 14).

During the meta-regression analysis of acupuncture versus sham-acupuncture, the disease course (adjusted $\mathrm{R}^{2}:-4.28 \%, \mathrm{I}^{2}$ resid: $90.81 \%, \mathrm{P}: 0.458$ ), age (adjusted $\mathrm{R}^{2}:-11.08 \%, \mathrm{I}^{2}$ resid: $90.84 \%, \mathrm{P}: 0.86$ ), baseline HAMD-24 (adjusted $\mathrm{R}^{2}:-9.92 \%, \mathrm{I}^{2}$ resid: $90.80 \%$, P: 0.35 ), and acupuncture session (adjusted $\mathrm{R}^{2}$ : $\left.-8.81 \%, \mathrm{I}_{\text {resid }}: 91.01 \%, \mathrm{P}: 0.643\right)$ were not statistically significant (Appendix 15).

No studies with significant differences were found in the sensitivity analyses of Acupuncture versus sham acupuncture (Appendix 16).

\subsubsection{Adverse Events}

Pooled data from 9 studies provided high-quality evidence that acupuncture (OR: 0.27 [95\% Cl: 0.12 to 0.61 ]; $\left.\right|^{2}=34 \%$ ) led to fewer adverse events compared to antidepressants (Table 1 and Figure 9) and low-quality evidence that acupuncture was not statistically different from sham-acupuncture (Table 1 and Appendix 17). Furthermore, low-quality evidence showed that acupuncture plus antidepressants were not statistically different from antidepressants (Table 1 and Appendix 18). 


\section{Discussion}

The systematic review included 43 high-quality RCTs involving 4037 MDD patients, while the meta-analysis included 41 RCTs with 3387 patients. The high quality evidence demonstrated an association between acupuncture with or without antidepressants and significant reduction in HAMD-24 scores. Importantly, relatively few adverse events were associated with acupuncture, consistent with previous studies and reviews ${ }^{8-1145}$. Our study provided the latest combined evidence for acupuncture alone or in combination with antidepressants in the treatment of MDD and identified research gaps that remain to be addressed.

Unlike previous systematic reviews, only high-quality RCTs were included in this study, ensuring that high-quality results were produced ${ }^{20-226970}$. Furthermore, the interventions in this study were restricted to acupuncture or acupuncture plus antidepressants, which greatly reduced the impact of diversiform acupuncture therapies ${ }^{71-74}$. Accordingly, the present meta-analysis found that acupuncture and acupuncture plus antidepressants were associated with significantly reduced HAMD-24 scores than sham acupuncture or antidepressants, which was not documented in previous reviews 226970 , which could be accounted for by our strict inclusion of high-quality RCTs ${ }^{8-1332-68}$. In addition, more stringent inclusion criteria were used to ensure the quality of the source of the included randomized controlled trials.

Positive results from sham acupuncture RCTs suggested that acupuncture in combination with antidepressants had more beneficial effects than sham acupuncture plus antidepressants. Importantly, sham acupuncture helped to exclude the placebo effect of acupuncture ${ }^{8}$. Insufficient blinding in included studies also increased the risk of bias of this meta-analysis. However, in recent years, pragmatic unblinded trials have been recommended to obtain clinically relevant results since they emphasized practical applicability in the real world and extrapolation (increased external validity) rather than treatment effects ${ }^{75}$. This design of our study was well suited to complex and flexible interventions despite the inability to blind acupuncturists ${ }^{75-77}$. Interestingly, it has also been suggested that placebo could play a role in the effects of acupuncture ${ }^{78-81}$, and pragmatic nonblinded trials could provide more useful evidence for clinical guidelines of acupuncture ${ }^{82}$. However, a gap still exists between the findings of RCTs of Acupuncture and our observations during clinical practice.

Clinically, acupuncture is often used to treat pain and plays an adjunctive treatment role in depression. The intended meaning is not clear. Did the author mean "The evidence from our study substantiated that acupuncture could be used as a major treatment approach for depression; however, the results of the heterogeneity analysis suggested that the results of data synthesis were not robust. Indeed, heterogeneity is an issue that cannot be avoided in metaanalysis $^{83}$, and in the present study, greater heterogeneity was found after data pooling. Importantly, a meta-regression analysis found an association between baseline HAMD scores and acupuncture treatment effectiveness, consistent with some previous studies ${ }^{170}$.

Therefore, we do not believe that Acupuncture is suitable as the sole treatment for depression. However, the latest clinical evidence suggests that acupuncture has good clinical effectiveness in improving HAMD scores and improving 5-HT and GABA levels ${ }^{32} 414868$. However, these studies had small sample sizes, emphasizing the need for high-quality studies with large samples to corroborate these findings.

\section{Limitations}

There were several limitations to this study. Significant heterogeneity was found in the results of this study, which lowered the level of evidence, while the subgroup analysis did not reduce heterogeneity. There are many similar treatment modalities for Acupuncture, and this study was unable to determine whether Acupuncture was the best treatment modality. The number of acupuncture treatments varied considerably in the included studies, but no association with effectiveness was found after meta-regression analysis. Accordingly, it was not possible to clarify the optimal number of acupuncture sessions for MDD. Funnel plots were not feasible for most outcomes due to the limited number of trials included while assessing each outcome in the meta-analysis, explaining why publication bias could not be fully assessed. Only one of the studies included in this review was not done in China; accordingly, the fact that Chinese people tend to believe in the therapeutic effects of acupuncture may be another source of bias.

\section{Conclusions}

The findings of this systematic review and meta-analysis provided high-quality evidence that acupuncture or acupuncture in combination with antidepressants significantly reduced HAMD-24 scores.

\section{Declarations}

\section{Patient and Public Involvement}

No patient was involved.

\section{Author contribution}

Conceptualization: Guixing Xu, Qiwei Xiao, Hanzhou Lei, Ling Zhao, Fanrong Liang.

Data curation: Hanzhou Lei, Guixing Xu, Biqing Huang, Zihan Yin, Qiwei Xiao. 
Formal analysis: Guixing Xu, Zihan Yin, Liuyang Huang, Qiwei Xiao.

Funding acquisition: Fanrong Liang, Ling Zhao.

Investigation: Hanzhou Lei, Jiao Chen.

Methodology: Guixing Xu, Zihan Yin.

Project administration: Fanrong Liang, Ling Zhao.

Resources: Liuyang Huang, Zhuo Zhou, Hao Tian.

Software: Guixing Xu, Biqing Huang.

Supervision: Fanrong Liang, Zihan Yin, Qiwei Xiao, Mingsheng Sun.

Validation: Hanzhou Lei, Guixing Xu.

Visualization: Guixing Xu.

Writing - original draft: Guixing Xu, Qiwei Xiao, Biqing Huang.

Writing - review \& editing: Guixing Xu, Qiwei Xiao.

\section{Funding}

This study has been supported by National Natural Science Foundation (No. 81590950, No.81590951), China. Funding sources had no role in the design of this study and will have no role during its execution, analysis, interpretation of data, or decision to present results.

\section{Availability of data and materials}

The findings of this meta-analysis and systematic review will be published in peer-reviewed publications or conference presentations, and all of the data will be reported.

\section{Competing interests}

None.

\section{Consent for publication}

Not applicable.

\section{Ethics approval and consent to participate}

Ethics approval and patient consent were not required since this is secondary research without patient involvement.

\section{Author details}

${ }^{1}$ Acupuncture and Tuina School, Chengdu University of Traditional Chinese Medicine, 37 Shi-er-qiao Rd, Chengdu 610075, Sichuan, China.

${ }^{2}$ Institute of Acupuncture and Moxibustion, China Academy of Chinese Medical Sciences, No. 3, Hai yun cang Hutong, Dong cheng District, Beijing 100007, China.

\section{Acknowledgements}

This review would like to acknowledge Home for Researchers \www.home-for-researchers.com『for language help.

\section{Patient consent}

Not required. 


\section{Provenance and peer review}

Not commissioned; externally peer reviewed.

\section{References}

1. Gartlehner G, Wagner G, Matyas N, et al. Pharmacological and non-pharmacological treatments for major depressive disorder: review of systematic reviews. BMJ open 2017;7(6)

2. Friedrich, M. J. Depression Is the Leading Cause of Disability Around the World. Jama 2017;317(15):1517.

3. Kearney M, Orrell RW, Fahey M, et al. Pharmacological treatments for Friedreich ataxia. Cochrane Database Syst Rev 2016;8(8):CD007791.

4. Claire C, Kerry G, John R, et al. Long-term antidepressant use: patient perspectives of benefits and adverse effects. Patient Preference and Adherence 2016;10:1401-07.

5. Thase ME, Koenig AM. Outcomes in the Treatment of Major Depressive Disorder. Chapter 2009:22-53.

6. Bannerman RH. The World Health Organization viewpoint on acupuncture. Am J Acupuncture 1980

7. Ebrary I. Acupuncture: Review and analysis reports on controlled clinical trials.

8. Almi A, Mihardja H, Srilestari A, et al. Therapeutic effect of acupuncture combined with antidepressants on changes in the HAMD-17 score in major depressive disorder. Journal of Physics: Conference Series 2018;1073 doi: 10.1088/1742-6596/1073/6/062037

9. Fan L, Gong J, Fu W, et al. Gender-Related Differences in Outcomes on Acupuncture and Moxibustion Treatment Among Depression Patients. Journal of alternative and complementary medicine (New York, NY) 2015;21(11):673-80. doi: 10.1089/acm.2015.0068 [published Online First: 2015/08/21]

10. Zhang WJ, Yang XB, Zhong BL. Combination of acupuncture and fluoxetine for depression: a randomized, double-blind, sham-controlled trial. Journal of alternative and complementary medicine (New York, NY) 2009;15(8):837-44. doi: 10.1089/acm.2008.0607 [published Online First: 2009/08/15]

11. Wenbing F, Li F, Xiaoping Z, et al. Clinical Research on Acupuncture Treatment of Depressive Neurosis by Using Liver-function-regulating Method. Acupuncture Research 2006(06):355-58.

12. Li J, Jianhua L, Jianpen $\mathrm{H}$, et al. Influence of acupuncture in Compare with sham acupuncture on hamd Factors in Mild and moderate depression. Chinese Archives of Traditional Chinese Medicine 2016;34(08):1916-19.

13. Jian L, Aiai W, Guangning N, et al. Acupuncture for female depression a randomized controlled trial. Chinese Acupuncture \& Moxibustion 2018;38(04):375-78.

14. Russowsky BA, Mariana L, Kaptchuk TJ, et al. Placebo Response of Non-Pharmacological and Pharmacological Trials in Major Depression: A Systematic Review and Meta-Analysis. PloS one 2009;4(3):e4824-

15. Napadow V, Makris N, Jing L, et al. Effects of electroacupuncture versus manual acupuncture on the human brain as measured by fMRI. Human brain mapping 2010;24(3):193-205.

16. Hou Z, Jiang W, Yin Y, et al. The Current Situation on Major Depressive Disorder in China: Research on Mechanisms and Clinical Practice. Neuroscience Bulletin 2016

17. Wang Z, Wang X, Jian L, et al. Acupuncture treatment modulates the corticostriatal reward circuitry in major depressive disorder. Journal of psychiatric research 2017;84:18-26.

18. Wang $\mathrm{Y}$, Jiang $\mathrm{H}$, Hong M, et al. Antidepressant Mechanism Research of Acupuncture: Insights from a Genome-Wide Transcriptome Analysis of Frontal Cortex in Rats with Chronic Restraint Stress. Evid-based Complement Altern Med 2017;2017:1-13.

19. Leung M, Yip KK, Ho YS, et al. Mechanisms Underlying the Effect of Acupuncture on Cognitive Improvement: A Systematic Review of Animal Studies. Journal of Neuroimmune Pharmacology 2014;9(4):492-507.

20. Guixing X, Juan L, Zihan Y, et al. Quality and applicability assessment for systematic reviews on acupuncture treatment for primary depression. CHINESE JOURNAL OF EVIDENCE-BASED MEDICINE 2019;19(11):1333-40.

21. Guixing X, Juan L, Zihan Y, et al. Acupuncture for Primary Depression: A Overview of Systematic Reviews. Journal of Basic Chinese Medicine 2021;27(01):107-13.

22. Li M, Niu J, Yan P, et al. The effectiveness and safety of acupuncture for depression: An overview of meta-analyses. Complementary therapies in medicine 2020;50:102202. doi: 10.1016/j.ctim.2019.102202 [published Online First: 2020/05/24]

23. Shea BJ, Reeves BC, Wells G, et al. AMSTAR 2: a critical appraisal tool for systematic reviews that include randomised or non-randomised studies of healthcare interventions, or both. The BMJ 2017;358:j4008.

24. Moher D, Shamseer L, Clarke M, et al. Preferred reporting items for systematic review and meta-analysis protocols (PRISMA-P) 2015 statement. Systematic reviews 2015;4(1):1-9.

25. Moher, David. Altman DG; PRISMA Group. Preferred reporting items for systematic reviews and meta-analyses: the PRISMA statement. PLoS medicine 2009;339(3)

26. Zeng XT, Xu C, Zhang C, et al. Implementation of Network Meta-Analysis Using Stata Soft ware. Chinese Journal of Evidence-Based Medicine 2013;13(11):1387-91.

27. Zou YZ, Cui JF, Han B, et al. Chinese psychiatrists views on global features of CCMD-III, ICD-10 and DSM-IV. Asian Journal of Psychiatry 2008;1(2):56-59. 
28. Do L. American Psychiatric Association Diagnostic and Statistical Manual of Mental Disorders (DSM-IV). Springer US 2011

29. Sheehan DV, Lecrubier Y, Sheehan KH, et al. The Mini-International Neuropsychiatric Interview (M.I.N.I.): the development and validation of a structured diagnostic psychiatric interview for DSM-IV and ICD-10. The Journal of clinical psychiatry 1998;59(Suppl 20):22-33.

30. Higgins J. Cochrane Handbook for Systematic Reviews of Interventions Version 5.0.1. The Cochrane Collaboration. Naunyn-Schmiedebergs Archiv für experimentelle Pathologie und Pharmakologie 2008;5(2):S38.

31. Meerpohl JJ, Langer G, Perleth M, et al. [GRADE guidelines: 3. Rating the quality of evidence (confidence in the estimates of effect)]. Zeitschrift für Evidenz Fortbildung und Qualität im Gesundheitswesen 2012;106(6):449-56.

32. Lai W, Zuyan Z, Dan L, et al. RCTs of Fuyang Yiyin Acupuncture in Treating Depression. Journal of Clinical Acupuncture and Moxibustion 2021;37(08):30-34.

33. Meiyun C. The Clinical Study of Using Acupuncture Based on Combination of Yuan and Luo points Method and Cognitive Therapy on Depression of the Liver qi Stagnation Syndrome [PhD]. Nanjing University of Chinese Medicine, 2014.

34. Huan L. Clinical study of old ten-" to treat Comorbid anxiety and depression [Master]. Chengdu University of Chinese Medicine, 2015.

35. Qianqian S. Clinical Study on Lingguibafa Combined with Music Therapy in the Treatment of the Depression of Liver and Qi Stagnation [Master]. Guangxi University of Chinese Medicine, 2020.

36. Qiaojun L. Clinical therapeutic effect of ping wan li zhi acupuncture manipulation in the treatment of depression due to the deficiency of heart and spleen [Master]. Yunnan University of Chinese Medicine, 2019.

37. Yueqing L. The Clinical Research on the Combination of Shallow Insertion and Fluoxetine Hydrochloride in Treating Depression with Liver-qi Stagnation Type [Master]. Fujian University of Chinese Medicine, 2014.

38. Mengmeng M, Junmei W. Clinical observation of "Shugan Jianpi, Tongdu Tiaoxinacupuncture in the treatment of liver qi stagnation type depression. Clinical Journal of Traditional Chinese Medicine 2015;27(08):1115-17.

39. Yan D, Qing X. Clinical observation of 60 cases of depression treated with acupuncture. Medical Information 2013(16):616-16.

40. Kexin L. THE CLINICAL OBSERVATION OF ACUPANETURE TREATMENT IN DEPRESSION [Master]. Heilongjiang University of Chinese Medicine, 2011.

41. Haixing L. Cl inical efficacy observation of acu-therapy of Bal anc ing and Adjust ing Ren and Du on mild to Moderate Depress ion patients with the syndrome of i iver-depress ion and spleen-deficiency [Master]. Guangzhou University of chinese Medicine, 2019.

42. The Clinical Study of "Tiao Shen Jie Yu"Acupuncture Treatment on Depression of Liver Qi Stagnation. WCCU's 3rd Academic Conference on Chinese Medicine and Integrative Medicine in Geriatrics; 2010; Nanyang, Henan, China.

43. Jinyan T. THE CLINICAL OBSERVATION ON THERAPEUTIC EFFECT OF REGULATING SPIRIT TO RELIEVE DEPRESSION ACUPUNCTURE THERAPY OF DEPRESSION (STAGNANCY OF LIVER QD) [Master]. Heilongjiang University of Chinese Medicine, 2010.

44. Junyi C, Guo'an D, Le H, et al. Clinical Study on acupuncture in Method of regulating mentality and Smoothing Liver Combined with SSRI. Journal of New Chinese Medicine 2018;50(12):191-93.

45. Yuanhao D, Guiping L, Hong Y, et al. Clinical study on needling method for regulating mental activities and soothing liver for treatment of melancholia. Chinese acupuncture \& Moxibustion 2005(03):7-10.

46. Chao L. The clinical research of comorbid anxiety and depression with acupuncture to regulate the heart and soothe the nerves [Master]. Chengdu University of Chinese Medicine, 2015.

47. Run Z. Cl inical Study on the appl icat ion of tongyuan theory to acupuncture and acupoint catgut embedd ing therapy on Depressive neurosis [Master]. Guangzhou Un i ver s i ty of chinese Medicine, 2015.

48. Aijia Z. Clinical Study on the Treatment of Mild and Moderate Depression with Acupuncture and Moxibustion for Warming Yang and Expelling Pathogenic Factors [Master]. Shanghai University of Chinese Medicine, 2019.

49. Yu S, Zhixiang W, Zheqi Z, et al. Clinical Study on Needling Method of Purging Liver and Tonifying Lungs in Treating Taiyin Cases with Depression. Journal of Nanjing University of Traditional Chinese Medicine 2015;31(2) [published Online First: 20161205]

50. Kai X. 120 cases of depression treated with acupuncture. Liaoning Journal of Traditional Chinese Medicine 2011;38(10):2060-62.

51. Li F, Wen-bin F, Chang-rong M, et al. Effect of acupuncture at routine acupoint and non-acupoint on depressive neurosis evaluated with Hamilton depression scale. Chinese Journal of Clinical Rehabilitation 2005(28):14-16.

52. Cheng L. a clinical study of synergy effect of acupuncture host points combined with local warm needling method in the treatment of depression of heart-spleen deficiency syndrome type [Master]. Yunnan University of Chinese Medicine, 2019.

53. Ling S. Clinical efficacy of acupuncture combined with biofeedback therapy in the treatment of depression [Master]. Guiyang College of Traditional Chinese Medicine, 2014.

54. Hai-dong C, Xiu-yan Y, Xue-hong M, et al. Effects of acupuncture in Patients with mild or Moderate depression treated with paroxetine hydrochloride. Chinese Journal of Information on Traditional Chinese Medicine 2014;21(08):35-38.

55. Yi L, Baoliang Z, Zhuowei H, et al. Acupuncture Therapy for Depressive and Anxious Symptoms of Outpatients Receiving methadone maintenance treatment: a Randomized Controlled tria. Chinese journal of clinical psychology 2012;20(03):363-66.

56. Li J. A Random i zed Control led Study on Acupuncture Treatment of Siguan" Points for Depression and the Ac-cAMP-PKA Postreceptor signal Transduction Mechan i sm [PhD]. Guangzhou University of Chinese Medicine, 2012.

Page 9/16 
57. Chuanwei C. Cl inical Observation on Treating Neurotic Depress ion between Fluoxetine and Acupuncture [Master]. Guangzhou University of Chinese Medicine, 2007.

58. Chong W. Study of clinical effect and improvement of tcM syndromes of acupuncture for depression with I iver-qi stagnation syndrome [Master]. Guangzhou University of Chinese Medicine, 2007.

59. Fenghe K, Hong L. 61 cases of depression treated with acupuncture. Chinese Journal of Gerontology 2013;33(10):2380-82.

60. Guanghai W. Clinical Study on Improving the Quality of Life of Patients with mild to Moderate Depression by Acupuncture and Moxibustion [PhD]. Guangzhou University of Chinese Medicine, 2017.

61. Jing Z, Yeqing D, Yuning W, et al. The efficacy of acupuncture and medicine in the treatment of mild to moderate depression. Shanghai Journal of Acupuncture and Moxibustion:1-6.

62. Juan X, Guo C, Lizhi O, et al. Clinical efficacy of acupuncture and moxibustion combined with gua sha in the treatment of depression evidence. Journal of Clinical Acupuncture and Moxibustion 2016;32(05):19-21.

63. Lizhi C. The Observations on Long-Term Curative Effects of the Combination of Acupuncture and medication in Depression Treatments [Master]. Nanjing University of Chinese Medicine, 2011.

64. Weirong L, Yong H, Junqi C, et al. Global improvement in agitated depression treated with the alliance therapy of acupuncture and seroxat and the observation of the quality of life. Chinese Acupuncture \& Moxibustion 2012;32(12):1063-69.

65. Wenbing F, Li F, Xiaoping Z, et al. Acupuncture for treatment of depressive neurosis a multi-center randomized controlled study. Chinese Acupuncture \& Moxibustion 2008(01):3-6.

66. Yan H. A CLINICAL OBSERVATION OF COMBINED ACUPUNCTURE AND MEDICINAL TREATMENT IN DEPRESSION SYNDROME P300 EFFECTS [Master]. Nanjing University of Chinese Medicine, 2010.

67. Yanhong L. Clinical Comparative Study of Electro-Acupuncture and Manual Acupuncture in Treating Depression [Master]. Nanjing University of Chinese Medicine, 2012.

68. Yijun C. Clinical observation on the Treatment of Mild and Moderate Depression by Zhuang heat-sensitive moxibustion acupuncture [Master]. Guangxi University of Chinese Medicine, 2020.

69. Armour M, Smith CA, Wang LQ, et al. Acupuncture for Depression: A Systematic Review and Meta-Analysis. Journal of clinical medicine 2019;8(8) doi: 10.3390/jcm8081140 [published Online First: 2019/08/03]

70. Smith CA, Armour M, Lee MS, et al. Acupuncture for depression. The Cochrane database of systematic reviews 2018;3(3):Cd004046. doi: 10.1002/14651858.CD004046.pub4 [published Online First: 2018/03/05]

71. Chen X-I, Xu K, Luo R-h, et al. Electroacupuncture in treating depression: A randomized controlled study. J Acupunct Tuina Sci 2013;11(1):42-45. doi: 10.1007/s11726-013-0653-X

72. Duan H, Honglin Z, Xiaoling W, et al. Comparative Analysis of Clinical Efficacy of Electro-acupuncture and Simple Acupuncture in the treatment of first-Episode mild-to-Moderate depression. J Tradit Chin Med 2019;60(15):1304-07.

73. Zhijun L, Qihua Y, Yang F, et al. Evaluating Clinical Curative Effect on the Treatment of the Depression With Liver's Qi Stasis by Magnetic-acupoint Therapy. Chinese Archives of Traditional Chinese Medicine 2009;27(12):2679-81.

74. Xiaodong X. esearch on moxibustion treatment of students depression in patients with health related quality of life and cognitive function [Master]. Chengdu University of Chinese Medicine, 2014.

75. Sox HC, Lewis RJ. Pragmatic Trials: Practical Answers to ?Real World? Questions. JAMA The Journal of the American Medical Association 2016;316(11):1205.

76. Ford I, Norrie J. Pragmatic Trials. New England Journal of Medicine 2016;375(5):454.

77. Chalkidou K, Tunis S, Whicher D, et al. The role for pragmatic randomized controlled trials (pRCTs) in comparative effectiveness research. Clinical Trials 2012;9(4):436-46.

78. Fan AY, Xu J, Li YM. Evidence and expert opinions: Dry needling versus acupuncture (II): The American Alliance for Professional Acupuncture Safety (AAPAS) White Paper 2016. Chinese journal of integrative medicine 2017;23(2):83-90. doi: 10.1007/s11655-017-2800-6 [published Online First: 2017/03/08]

79. Gong CZ, Liang FR, Li CH, et al. [Discussion on Acupuncture as adjunctive therapy for chronic stable angina: a randomized clinical trial published in JAMA Internal Medicine]. Zhongguo zhen jiu = Chinese acupuncture \& moxibustion 2021;41(4):359-64. doi: 10.13703/j.0255-2930.20190929-k0003 [published Online First: 2021/04/29]

80. Li YM. [Puzzles and hypotheses of acupuncture]. Zhongguo Zhong xi yi jie he za zhi Zhongguo Zhongxiyi jiehe zazhi = Chinese journal of integrated traditional and Western medicine 2013;33(11):1445-8. [published Online First: 2014/02/04]

81. Wang H, Yang G, Wang S, et al. The Most Commonly Treated Acupuncture Indications in the United States: A Cross-Sectional Study. The American journal of Chinese medicine 2018:1-33. doi: 10.1142/s0192415x18500738 [published Online First: 2018/10/10]

82. Kaptchuk TJ, Keji C, Jun S. Recent Clinical Trials of Acupuncture in the West: Responses from the Practitioners. Chinese Journal of Integrative Medicine: English Edition 2010;000(003):P.197-203.

83. JPT H. Cochrane Handbook for Systematic Reviews of Interventions Version 5.1.0 [updated March 2011]. In: S G, ed. The Cochrane Collaboration. http://handbook.cochrane.org, 2011.

Page $10 / 16$ 

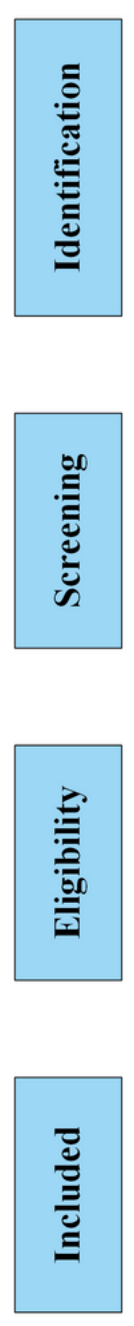

Figure 1

Flowchart of study selection

\begin{tabular}{|c|c|c|c|c|c|c|c|}
\hline \multirow[b]{2}{*}{ Study or Subgroup } & \multicolumn{3}{|c|}{ Experimental } & \multicolumn{3}{|c|}{ Control } & \multirow[b]{2}{*}{ Weight } \\
\hline & Mean & SD & Total & Mean & SD & Total & \\
\hline Fan_2005a & -9.49 & 6.19 & 28 & -5.28 & 5.45 & 25 & $8.8 \%$ \\
\hline Fan_2015a & -13.44 & 5.07 & 47 & -6.24 & 4.28 & 48 & $11.9 \%$ \\
\hline Fan_2015b & -13.44 & 5.07 & 47 & -11.04 & 4.91 & 48 & $11.6 \%$ \\
\hline Fu_2006a & -16.76 & 4.38 & 78 & -8.83 & 6.95 & 41 & $10.7 \%$ \\
\hline Fu_2008a & -13.62 & 5.25 & 176 & -7.77 & 5.82 & 88 & $13.0 \%$ \\
\hline Jiang_2012a & -12.55 & 4.06 & 38 & -10.7 & 5.68 & 34 & $10.8 \%$ \\
\hline Jiang_2012b & -12.55 & 4.06 & 38 & -8.38 & 6.42 & 36 & $10.4 \%$ \\
\hline Jiang_2016a & -11.31 & 5.25 & 36 & -9.13 & 3.81 & 39 & $11.4 \%$ \\
\hline Jiang_2016b & -11.31 & 5.25 & 36 & -7.03 & 3.91 & 37 & $11.3 \%$ \\
\hline Total $(95 \% \mathrm{Cl})$ & & & 524 & & & 396 & $100.0 \%$ \\
\hline $\begin{array}{l}\text { Heterogeneity: } \mathrm{Tau}^{2} \\
\text { Test for overall effec }\end{array}$ & $\begin{array}{l}\text { 63; Chi } \\
=6.09\end{array}$ & 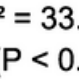 & $\begin{array}{l}1, \mathrm{df}= \\
0001)\end{array}$ & & & & $\%$ \\
\hline
\end{tabular}

9943 of records after duplicates removed

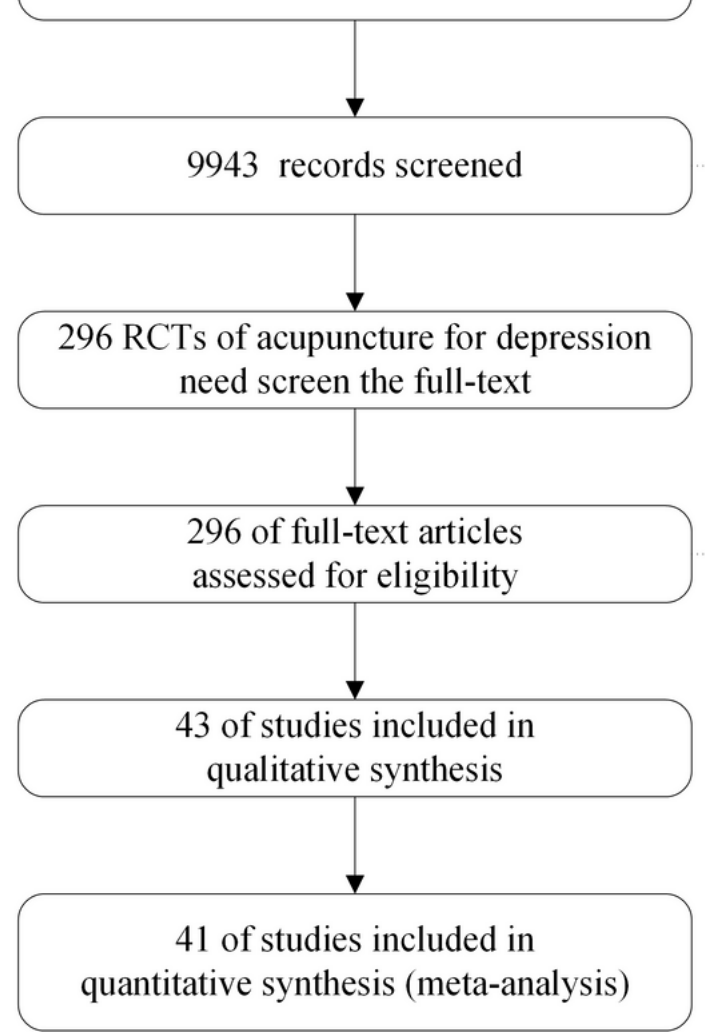

9360 of records excluded with following reason: non-RCTs: 1496; nonacupuncture: 533 ; nonexperiment: 1039 . depression: 6579; animal

\section{3 of full-text articles excluded (Jadad score $<4)$}

\section{Figure 2}




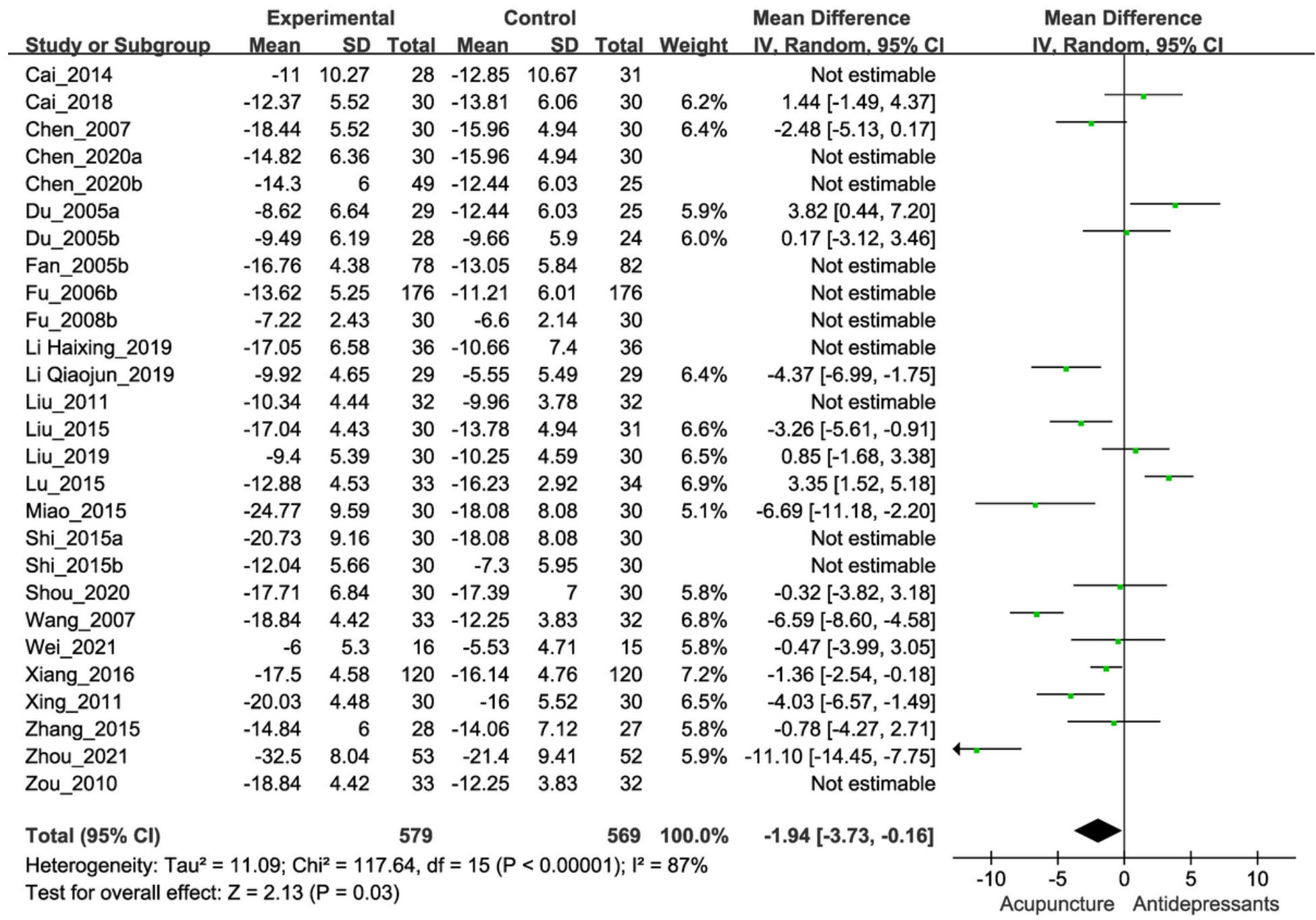

Figure 3

Forest plot of HAMD-24 for Acupuncture versus antidepressants 


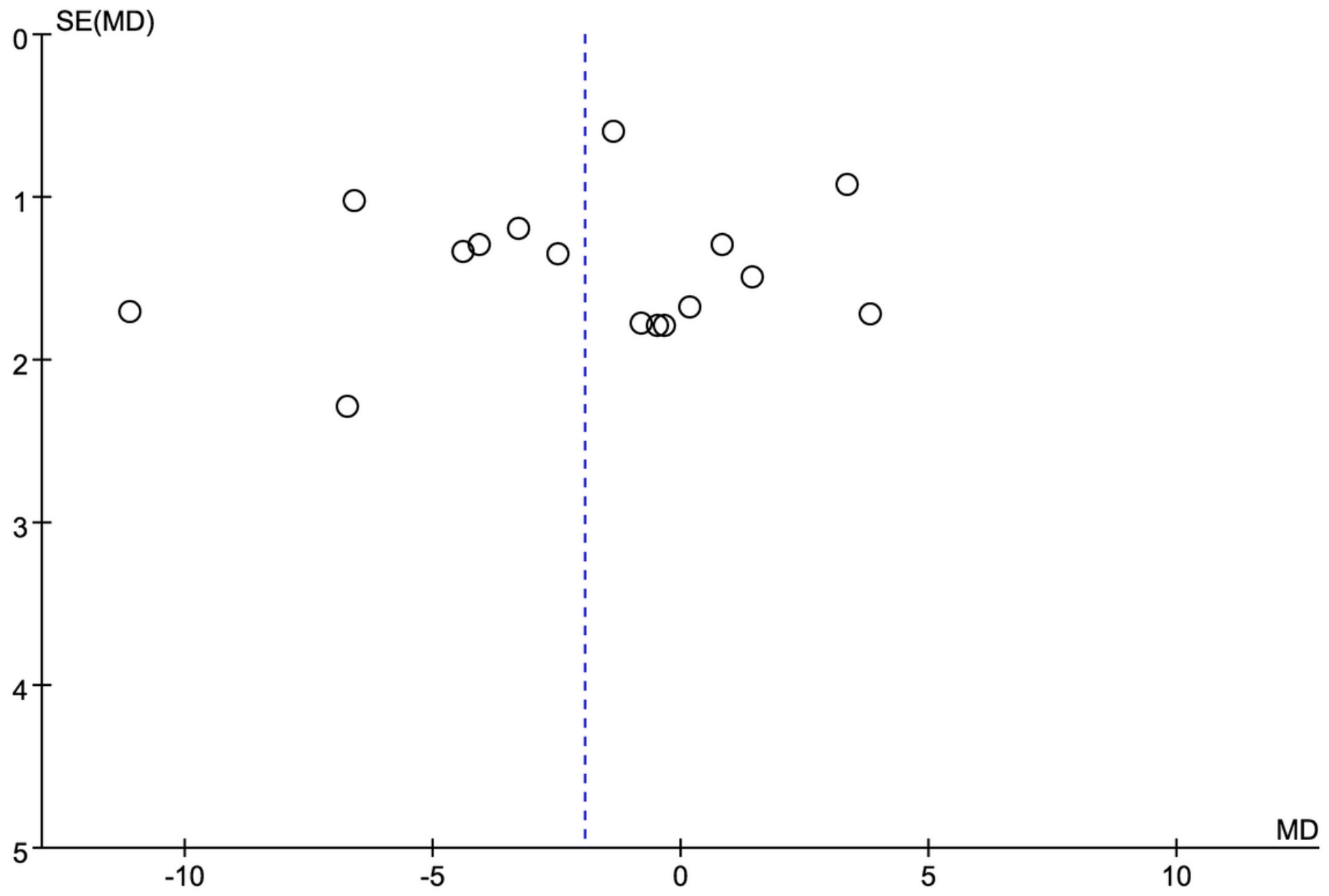

Figure 4

Funnel plot of HAMD-24 for Acupuncture versus antidepressants

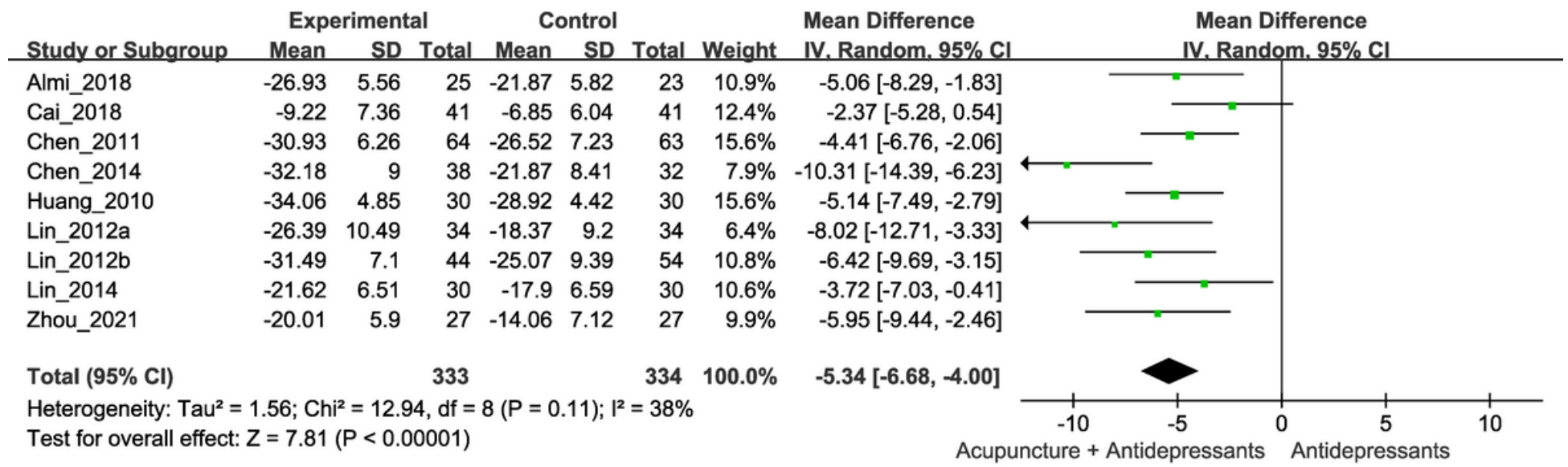

Figure 5

Forest plot of HAMD-24 for Acupuncture + antidepressants versus antidepressants 


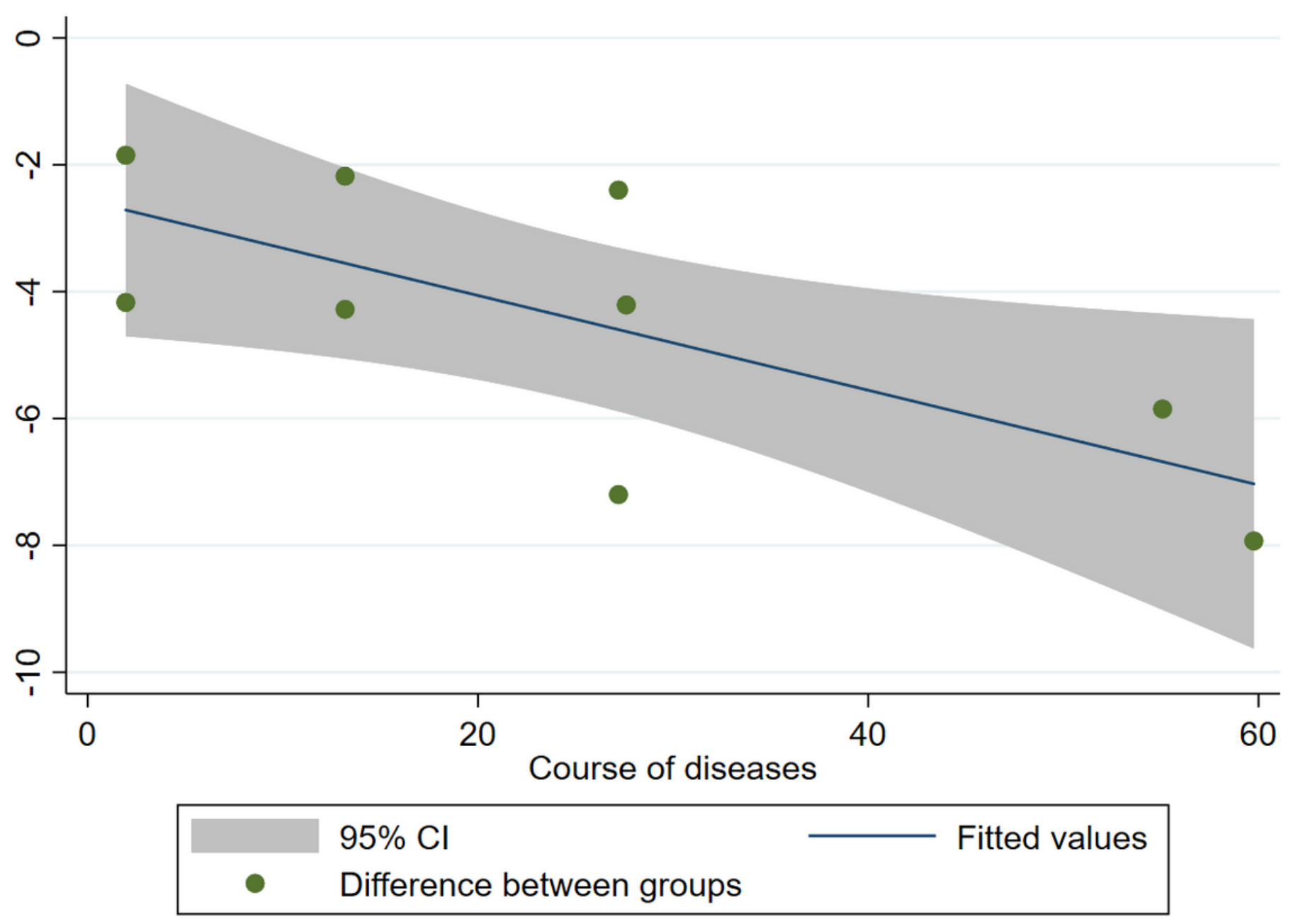

Figure 6

Meta-regression with baseline disease course as a single continuous covariate 


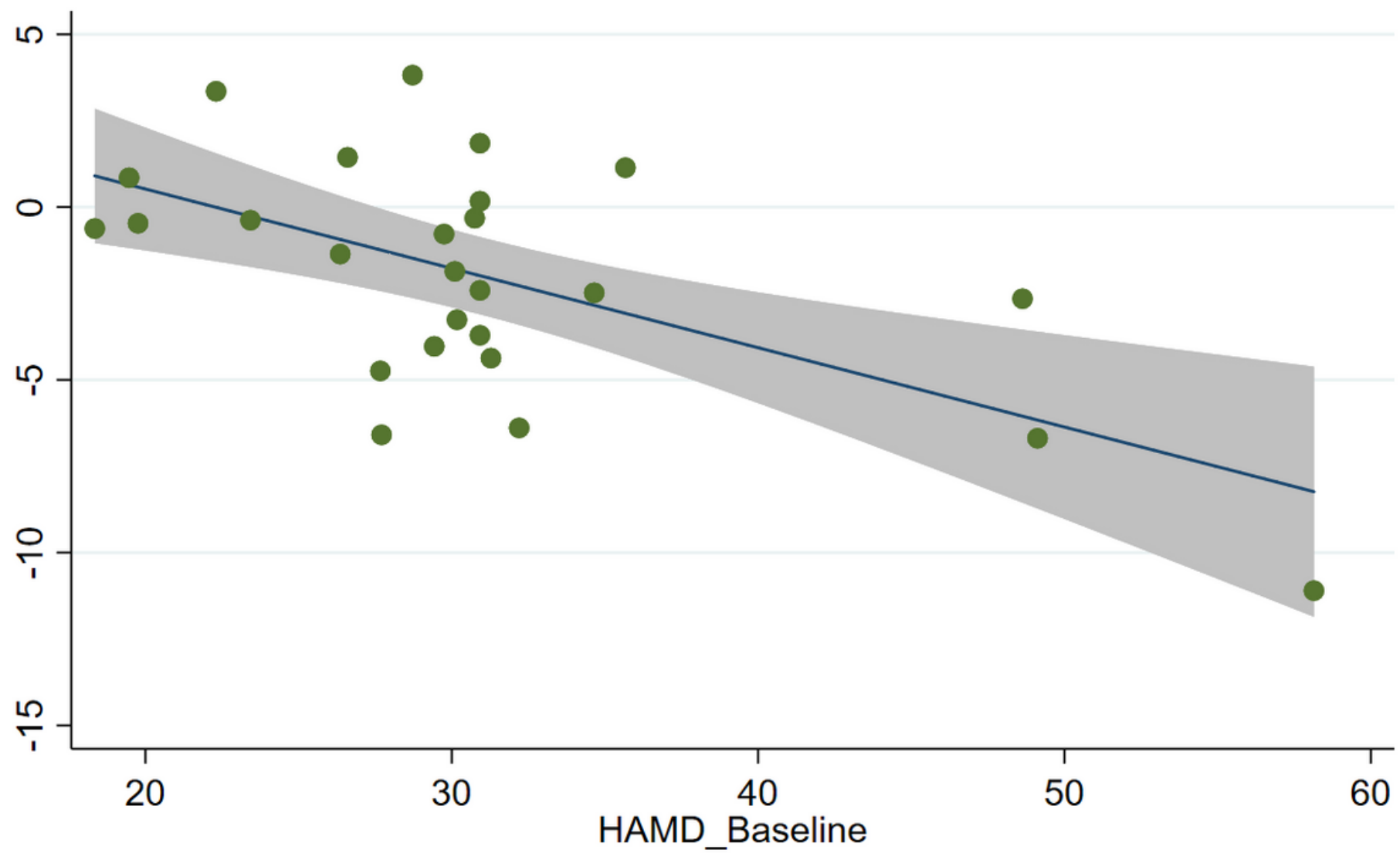

Figure 7

Meta-regression with baseline HAMD-24 as single continuous covariate

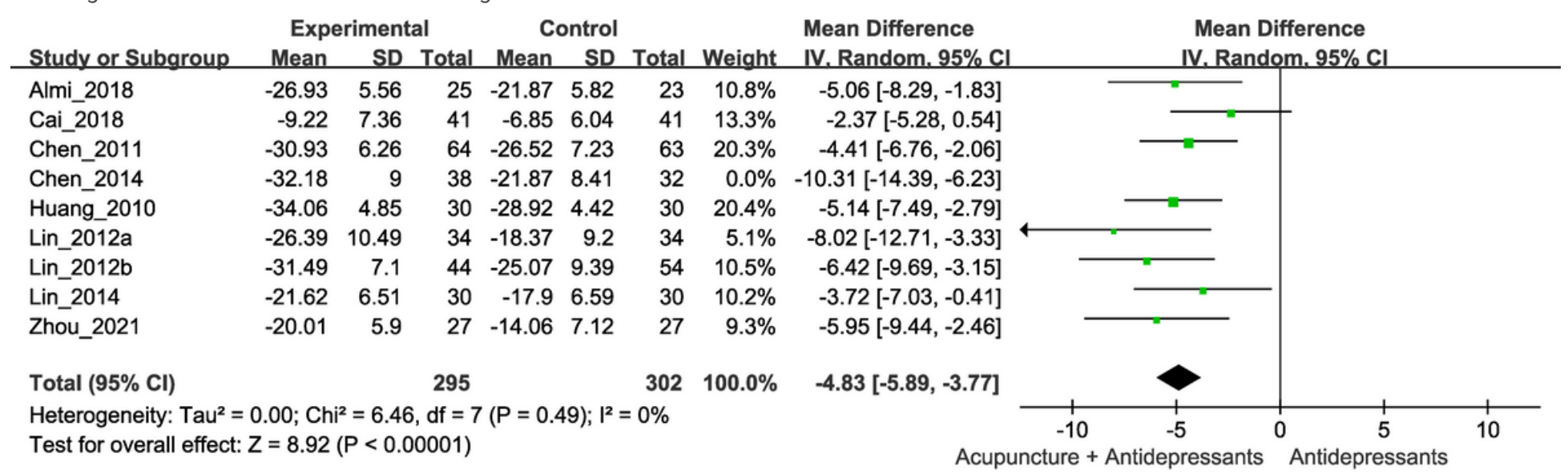

Figure 8

Forest plot of HAMD-24 for Acupuncture versus antidepressants with Chen_2014 removed 
Experimental Control Study or Subgroup

Chen_2007

Du_2005a

Du_2005b

Fan_2005a

Lu_2015

Miao_2015

Wang_2007

Wei_2021

Xiang_2016

Xiang_2016b

Total $(95 \% \mathrm{CI})$

Total events

Heterogeneity: $\mathrm{Tau}^{2}=0.53 ; \mathrm{Ch}^{2}=13.61, \mathrm{df}=9(\mathrm{P}=0.14) ; \mathrm{I}^{2}=34 \%$

Test for overall effect: $Z=3.16(P=0.002)$

Figure 9

Forest plot of adverse events for Acupuncture versus antidepressants

\section{Supplementary Files}

This is a list of supplementary files associated with this preprint. Click to download.

- AppendixV1.0.docx

- PRISMAChecklist.doc
Odds Ratio

Odds Ratio

Events Total Events Total Weight $\mathrm{M}-\mathrm{H}$, Random, 95\% Cl

$\begin{array}{lllll}3 & 30 & 10 & 30 & 16.0 \%\end{array}$

$\begin{array}{lllll}0 & 49 & 2 & 25 & 5.6 \%\end{array}$

$\begin{array}{lllll}0 & 29 & 2 & 25 & 5.6 \%\end{array}$

$\begin{array}{lllll}0 & 28 & 4 & 24 & 5.9 \%\end{array}$

$\begin{array}{lllll}1 & 30 & 7 & 30 & 9.6 \%\end{array}$

$\begin{array}{lllll}2 & 33 & 4 & 34 & 12.5 \%\end{array}$

$\begin{array}{lllll}6 & 30 & 20 & 30 & 18.9 \%\end{array}$

$\begin{array}{lllll}2 & 32 & 3 & 33 & 11.7 \%\end{array}$

$\begin{array}{lllll}6 & 15 & 0 & 15 & 5.9 \%\end{array}$

$\begin{array}{lllll}1 & 16 & 3 & 15 & 8.3 \%\end{array}$

292

$261 \quad 100.0 \%$ 55

$0.22[0.05,0.91]$

$0.09[0.00,2.06]$

$0.16[0.01,3.48]$

$0.08[0.00,1.57]$

$0.11[0.01,0.99]$

$0.48[0.08,2.84]$

$0.13[0.04,0.40]$

$0.67[0.10,4.28]$

$21.21[1.07,420.80]$

$0.27[0.02,2.90]$

$0.27[0.12,0.61]$
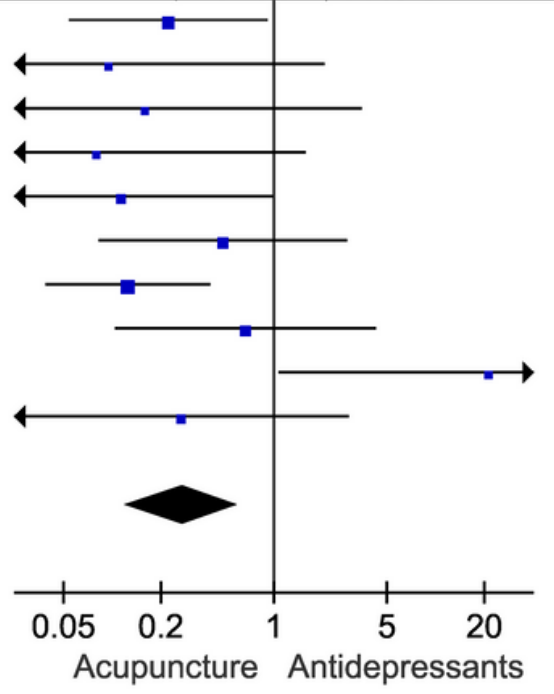